\title{
Developing an Electronic Search Strategy Algorithm for Detecting Professional Interpreter Use among Patients with Limited English Proficiency: A Derivation and Validation Study
}

\author{
Jalal Soleimani ( $\sim$ soleimani.jalal@mayo.edu ) \\ Mayo Clinic \\ Alberto Marquez \\ Mayo Clinic \\ Timothy Weister \\ Mayo Clinic \\ Amelia Barwise \\ Mayo Clinic
}

\section{Research Article}

Keywords: Limited English Proficiency, Electronic Health Record, Search Strategy, Algorithm, Derivation and Validation, Manual Review

Posted Date: December 21st, 2020

DOl: https://doi.org/10.21203/rs.3.rs-126974/v1

License: (c) (i) This work is licensed under a Creative Commons Attribution 4.0 International License.

Read Full License 


\section{Abstract \\ Background}

Evidence exists that disparities occur for patients with Limited English Proficiency (LEP) that impact the quality of medical services, outcomes, and patient satisfaction. Using interpreter services can reduce these negative impacts; therefore optimizing our understanding of interpreter use during patient care is important. Manual chart review is time-consuming. The objective of this study was to develop and validate a search strategy algorithm to detect patients who used professional interpreter services during their hospitalization.

\section{Methods}

We identified all adults who were admitted to the hospital who had at least one Intensive Care Units (ICU) admission during the hospital stay across the Mayo Clinic Enterprise between January 1, 2015, and June 30,2020 . Three random subsets of 100 patients were extracted from 60,268 patients admitted to an ICU

to develop the search strategy algorithm. A physician reviewer conducted the gold standard manual chart review and these results were compared with the search strategy algorithm each time it was refined. Iterative modification of the search strategy was performed and sensitivity and specificity were calculated by comparing the results to the reference standard for both derivation cohorts and the final validation cohort. Any uncertainties were resolved by a second physician researcher.

\section{Results}

The first search strategy resulted in a specificity of $95.7 \%$ and a sensitivity of $93.5 \%$. The second revised search strategy achieved a specificity of $96.7 \%$ and a sensitivity of $92.3 \%$. The final version of the search strategy was applied to the validation subset and specificity and sensitivity were $92.6 \%$ and $100 \%$ respectively.

\section{Conclusion}

We successfully derived and validated a search strategy algorithm to assess interpreter use among hospitalized patients. Developing a search strategy algorithm with a high sensitivity can reduce the time required to abstract data from the medical record compared to manual chart review. This can be used to examine and understand patient needs for research and quality improvement initiatives.

\section{Background}

The number of residents in the United States with Limited English Proficiency (LEP) has grown in recent decades.[1] According to the 2017 US Census, more than 64 million people aged 5 years and older speak 
a language other than English at home, and more than 25 million of the US population are classified as "speaking English less than very well" or having LEP.[2, 3] Furthermore approximately 60,000 to 85,000 patients travel to prestigious medical centers in the US every year for treatment potentially not available in their native countries and these patients also frequently have LEP.[4, 5]

Disparities in access, outcomes, and healthcare quality have been documented for patients with LEP.[614] Despite a national mandate for interpreter services to be deployed when patients with LEP are navigating the health care system, interpreters are underused, and integrating medical interpreting standards into clinical practice has been challenging.[15-17] Interpreter services positively impact communication and outcomes, increasing patient and family satisfaction with care, increasing understanding of diagnosis and treatment plans, reducing complications and length of hospital stay, and bolstering the use of preventive healthcare services.[11-13, 18-20] Across the lifespan from pediatric care to end of life care, interpreters have been shown to benefit patients.[14, 21]

Despite evidence of the benefit of interpreters, interpreters are underused as clinicians try to "get-by" with their own limited language skills or those of family members.[15, 22-24] The decision to use staff with limited language skills and family members instead professional interpreters may be influenced by access and availability of professional interpreter services based on organizational structures and resources.[25] Professional interpreters provide higher quality interpretation than family members and should be used.[26, 27]

It is important to identify when interpreters are needed and when interpreters are used, so we can improve our understanding of interpreter use and optimize systems for increasing timely interpreter use for patients who need an interpreter but may not get one. This knowledge will be a useful step towards sustained change to address disparities among those with LEP. The adoption of electronic health records (EHR) in place of traditional paper charts has provided unprecedented amounts of information (Big data) that allows researchers to evaluate larger cohorts of patients than traditional research approaches.[28, 29] Harnessing the potential of the EHR and the vast amounts of data within is challenging but developing and applying automated search strategies is a helpful approach. By using these tools to identify when an interpreter is utilized we can support quality improvement initiatives, enhance clinical practice, and improve our research accuracy and efficiency.

The objective of this derivation and validation study was to develop and validate a search strategy algorithm to detect patients that used professional interpreter services during their hospital stay. Although within demographic information "interpreter needed" may be recorded in the EHR, this does not necessarily mean an interpreter was used and identifying this more accurately and efficiently was the objective of this study.

\section{Methods}

This is a derivation and validation cohort study which was approved by the Mayo Clinic Institutional Review Board (IRB). We only included the data of those who gave prior research authorization in 
accordance with Minnesota state statutes if applicable.[30]

\section{Study population:}

We included consecutive adult patients ( $\geq 18$ years) who were admitted to the Intensive Care Units (ICU) across the Mayo Clinic enterprise (Minnesota, Wisconsin, Arizona, and Florida) over a period of $5 \frac{1}{2}$ years between January 1, 2015, and June 30, 2020. Mayo Clinic enterprise has 23 ICUs (14 in Minnesota, 4 in Florida, 3 in Wisconsin, and 2 in Arizona). The total cohort included 60,268 patients admitted to the hospital who had at least one ICU admission during the hospital stay. If a patient was admitted several times during this period, only the first admission including an ICU stay was included.

Our search strategy was designed to detect those using professioal interpreter sevices such as phone, video, or in-person interpreters, but not patients who used family, friends or healthcare team members as an interpreter. Three random subsets of 100 patients were used for the derivation and validation phases in keeping with previous studies conducted by our group and to ensure the time and effort required for manual chart review was feasible for the primary physician researcher reviewer.

\section{Manual data extraction strategies (Reference Gold Standard):}

Before developing the automated search strategy and to formulate the gold standard for interpreter use, members of the study team (JS, AB, AM) manually reviewed the EHRs of random patients classified as having a preferred language other than English and those patients whose charts indicated that an interpreter was required in demographic information. The study team examined the different ways that the" interpreter used" was documented by health care providers in the EHR including within flowsheets, and progress and encounter notes.

During the derivation and validation process, the reference gold standard comparison involved manual chart review of demographics, flowsheets, all clinical notes, and historical views by one physician researcher reviewer with a second reviewer resolving any uncertainty. Since clinical reasoning was not required, we felt this approach was reasonable for the development of this search strategy.

\section{Automated electronic search strategy:}

This retrospective cohort study used the interpreter indicator search in the ICU DataMart. DataMart is an extensive data warehouse containing a near-real-time normalized replica of Mayo Clinic's EHR. [31] DataMart contains patient demographic characteristics, diagnoses, laboratory results, and clinical flow sheets, gathered from various sources within the institution. ${ }^{(31)}$ The data within DataMart has been validated and is reliable.[31-33]

We also used an interpreter indicator search in Mayo Clinic's Advanced Cohort Explorer (ACE), an electronic retrieval query database within Mayo Clinic's Unified Data Platform (UDP). ACE is a powerful web-based software toolset that provides access to millions of patients charts and also enables the 
search of the EHR by specific text phrases or terms in specific parts of clinical notes. All data extracted by ACE can be exported to Excel to enable further statistical analysis.[28]

Derivations and validation were performed using three subset sample data. Each subset sample contained 100 patients that were randomly selected from the DataMart. (Fig. 1) These cohorts consisted of both those likely to use interpreter services and those not likely to use interpreter services. In order for patients to be categorized as "Interpreter used" using the automated search strategy, the patients needed to have both an interpreter indicator "Yes" within their demographic information as well as an applicable flowsheet articulating than an interpreter was used at the time of the encounter we were examining.

Following manual review of each of the derivation subsets, AM refined the electronic search strategy through several iterations of evaluation and refinement of the electronic search algorithm in the derivation cohorts. Once the search strategy was assessed and modified in the derivation cohorts, it was validated in the third cohort.

\section{Statistical analysis:}

An overall percent agreement between the electronic search algorithm and the manual medical record review was calculated. Sensitivity and specificity were calculated by comparing the results to the reference standard for each derivation and validation subsets. JMP statistical software (version 10.0.0; SAS Institute Inc., Cary, NC) was used for all analyses.

\section{Results}

The cohort of those with hospital admissions and at least one ICU admission across the Mayo Clinic Enterprise between January 1, 2015, and June 30, 2020, was 60,268 admissions. We excluded patients age less than 18 years, those who used a sign language interpreter, interpretation by family and friends, and those with no research authorization.

In the first derivation cohort, our manual review resulted in a specificity of $95.7 \%$ and a sensitivity of 93.5\%. The supervised algorithm was used for the second derivation subset. The second derivation, achieved a specificity of $96.7 \%$ and a sensitivity of $92.3 \%$. The final version of the search strategy for interpreter use was applied to the validation subset and this achieved a specificity of $92.6 \%$ and a sensitivity of $100 \%$ respectively. (Table 1 )

Table 1

Sensitivities and specificities of subset groups

\begin{tabular}{|c|c|c|}
\hline & Sensitivity (\%) & Specificity (\%) \\
\hline 1st Derivation & 93.5 & 95.7 \\
\hline 2nd Derivation & 92.3 & 96.7 \\
\hline Validation & 100 & 92.6 \\
\hline
\end{tabular}

Page 5/12 


\section{Discussion}

Currently, almost all healthcare institutions in the United States have adopted an EHR to integrate distributed data sources.[34-36] The large amount of data (big data) within the EHR presents great potential if strategies to examine it can be developed. Otherwise, it may present barriers to clinical research.[37, 38] Accessing big data usefully through the EHR and abstracting needed information for clinical research using manual data extraction methods is time-consuming and inefficient.

The purpose of this study was to develop an electronic automated search algorithm to reduce the time and effort needed to reliably identify patients who used interpreter services during their hospitalization. It is important to better understand professional interpreter use to foster strategies to increase timely and appropriate interpreter use in near-real time. Without strategies to increase the use of interpreter services, disparities for patients with LEP may get prepetuated. Interpreters reduce cultural, language, and literacy barriers with the goal of improving communication between patients and clinicians.[39]

In the present study, we developed an electronic search strategy with a high degree of sensitivity and specificity. The algorithm is more efficient than manual chart review for detecting patients who used interpreters in a large dataset, increasing our ability to abstract data accurately and quickly. This is useful for research and can improve research quality. The time to conduct manual chart review to identify if an interpreter has been used by a patient is about 10 minutes for a specific encounter. The developed search algorithm is able to identify the interpreter utilization within a large target group in a short time with $100 \%$ sensitivity. The results of this study are in line with other published studies emphasizing the benefit of electronic search strategies in big dataset extraction.[29, 40]

Strengths: This method of electronic data extraction by automated algorithm through institutional software connected to EHR is cost-effective, fast, and accurate compared to manual chart review. The total time frame for the physician researcher JS to conduct manual chart reviews of each set of 100 patients was about 15 hours. In contrast, the time needed to deploy the validated search strategy algorithm on a large study cohort usually takes between one and two hours. Hence, it is assumed that data extraction for interpreter use and anticipating the future need and preparation for service will consume less time in the future. Moreover, this study was performed on data across Mayo from multicenter ICUs, increasing our ability to generalize our results across the enterprise. We wanted to focus on professional interpreter use as there is evidence that unless absolutely necessary in an emergency situation,interpretation by family and friends should be avoided as accuracy and completeness of interpretation may be sub-optimal.[41-45]

Limitations: We were able to leverage institutional software and specific datasets (ACE and DataMart) to conduct our study. Other institutions with diverse computational infrastructure may need to modify this search strategy within their patient datasets and EHRs. Therefore external validation of this search strategy validation is needed. Additionally, the retrospective design of this study is a limitation for further validation prior to use as a predictor of interpreter needs. Despite the high sensitivity, the specificity of the 
validation subset decreased when compared to the first and second derivation subsets increasing the possibility of false-negative results.

\section{Conclusions}

We have successfully derived and validated an "Interpreter used" search strategy that identifies if a patient used a professional in-person interpreter, video-linked interpreter or telephone interpreter. It can be the deployed in the EHR demonstrating 100\% sensitivity and high specificity. This approach can substantially reduce the time commitment necessary to identify patients who used an interpreter compared with manual chart review of the EHR.

\section{Abbreviations}

Limited English Proficiency (LEP)

Intensive Care Units (ICU)

Electronic Health Records (EHR)

Institutional Review Board (IRB)

Advanced Cohort Explorer (ACE)

Unified Data Platform (UDP)

\section{Declarations}

Ethics approval and consent to participate: All methods were approved by the Mayo Clinic Institutional Review Board and performed in according with the relevant guidelines.

Consent for publication: All subjects were 18 and older and informed consent was obtained from all subjects.

Availability of data and materials: The datasets generated and/or analyzed during the current study are not publicly available due the Mayo clinic policy to protect patients information but are available from the corresponding author on reasonable request.

Competing interests: The authors declare that they have no competing interests.

Funding: This work was supported by the Division of Critical Care Medicine with no direct financial support.

Authors' contributions: 
$A B$ as a principal investigator designed the study, and data interpretation.

JS helped designed the study, performed data acquisition, and data interpretation manuscript writing.

AM designed the search algorithm and provided statistical analysis.

TW helped designed the study, designed the search algorithm, and provided statistical analysis.

All authors drafted the manuscript and/or revised it critically for important intellectual content and gave final approval of manuscript with all the accountability herein. All authors read and approved the final manuscript.

Acknowledgements: Not applicable

\section{References}

1. Pandya C, McHugh M, Batalova J. Limited English Proficient Individuals in the United States: Number, Share, Growth, and Linguistic Diversity. LEP Data Brief. Migration Policy Institute. 2011.

2. Bureau UC. Selected social characteristics in the United States: 2011-2015 American Community Survey 5-year estimates. https://www.census.gov/programs-surveys/acs/technicaldocumentation/table-and-geography-changes/2015/5-year.html; Accessed: September 02, 2020.

3. Patel AT, Lee BR, Donegan R, Humiston SG. Length of Stay for Patients With Limited English Proficiency in Pediatric Urgent Care. Clin Pediatr (Phila). 2020;59(4-5):421-8.

4. Ehrbeck T, Guevara C, Mango PD. Mapping the market for medical travel. The McKinsey Quarterly. 2008;11.

5. Van Dusen A. US hospitals worth the trip. Forbes com, Retrieved. 2008.

6. Lu T, Myerson R. Disparities in Health Insurance Coverage and Access to Care by English Language Proficiency in the USA, 2006-2016. J Gen Intern Med. 2020;35(5):1490-7.

7. Ridgeway JL, Njeru JW, Breitkopf CR, Mohamed AA, Quirindongo-Cedeno O, Sia IG, et al. Closing the Gap: Participatory Formative Evaluation to Reduce Cancer Screening Disparities among Patients with Limited English Proficiency. J Cancer Educ. 2020.

8. John-Baptiste A, Naglie G, Tomlinson G, Alibhai SM, Etchells E, Cheung A, et al. The effect of English language proficiency on length of stay and in-hospital mortality. Journal of general internal medicine. 2004;19(3):221-8.

9. Karliner LS, Kim SE, Meltzer DO, Auerbach AD. Influence of language barriers on outcomes of hospital care for general medicine inpatients. Journal of hospital medicine. 2010;5(5):276-82.

10. Betancourt JR, Renfrew M, Green A, Lopez L, Wasserman M. Improving patient safety systems for patients with limited English proficiency: a guide for hospitals. Rockville, MD: Agency for Healthcare Research and Quality. 2012:12-0041. 
11. Flores $G$. The impact of medical interpreter services on the quality of health care: a systematic review. Med Care Res Rev. 2005;62(3):255-99.

12. Jacobs EA, Shepard DS, Suaya JA, Stone E-L. Overcoming language barriers in health care: costs and benefits of interpreter services. American journal of public health. 2004;94(5):866-9.

13. Bagchi AD, Dale S, Verbitsky-Savitz N, Andrecheck S, Zavotsky K, Eisenstein R. Examining effectiveness of medical interpreters in emergency departments for Spanish-speaking patients with limited English proficiency: results of a randomized controlled trial. Ann Emerg Med. 2011;57(3):24856 e1-4.

14. Flores G, Abreu M, Barone CP, Bachur R, Lin H. Errors of medical interpretation and their potential clinical consequences: a comparison of professional versus ad hoc versus no interpreters. Ann Emerg Med. 2012;60(5):545-53.

15. Diamond LC, Schenker Y, Curry L, Bradley EH, Fernandez A. Getting by: underuse of interpreters by resident physicians. J Gen Intern Med. 2009;24(2):256-62.

16. Jacobs EA, Diamond LC, Stevak L. The importance of teaching clinicians when and how to work with interpreters. Patient education and counseling. 2010;78(2):149-53.

17. Lor M, Bowers BJ, Jacobs EA. Navigating Challenges of Medical Interpreting Standards and Expectations of Patients and Health Care Professionals: The Interpreter Perspective. Qual Health Res. 2019;29(6):820-32.

18. Barwise AK, Nyquist CA, Suarez NRE, Jaramillo C, Thorsteinsdottir B, Gajic O, et al. End-of-Life Decision-Making for ICU Patients With Limited English Proficiency: A Qualitative Study of Healthcare Team Insights. Critical care medicine. 2019;47(10):1380-7.

19. Jacobs EA, Lauderdale DS, Meltzer D, Shorey JM, Levinson W, Thisted RA. Impact of interpreter services on delivery of health care to limited-English-proficient patients. Journal of general internal medicine. 2001;16(7):468-74.

20. Chen Y, Criss SD, Watson TR, Eckel A, Palazzo L, Tramontano AC, et al. Cost and Utilization of Lung Cancer End-of-Life Care Among Racial-Ethnic Minority Groups in the United States. Oncologist. 2020;25(1):e120-e9.

21. Silva MD, Genoff M, Zaballa A, Jewell S, Stabler S, Gany FM, et al. Interpreting at the End of Life: A Systematic Review of the Impact of Interpreters on the Delivery of Palliative Care Services to Cancer Patients With Limited English Proficiency. Journal of Pain and Symptom Management. 2016;51(3):569-80.

22. Parsons JA, Baker NA, Smith-Gorvie T, Hudak PL. To 'Get by'or 'get help'? A qualitative study of physicians' challenges and dilemmas when patients have limited English proficiency. BMJ open. 2014;4(6).

23. Kale E, Syed HR. Language barriers and the use of interpreters in the public health services. $A$ questionnaire-based survey. Patient education and counseling. 2010;81(2):187-91.

24. López L, Rodriguez F, Huerta D, Soukup J, Hicks L. Use of interpreters by physicians for hospitalized limited English proficient patients and its impact on patient outcomes. Journal of general internal 
medicine. 2015;30(6):783-9.

25. Hsieh E. Not just "getting by": factors influencing providers' choice of interpreters. Journal of general internal medicine. 2015;30(1):75-82.

26. Gray B, Hilder J, Stubbe M. How to use interpreters in general practice: the development of a New Zealand toolkit. Journal of Primary Health Care. 2012;4(1):52-61.

27. Gray B, Hilder J, Donaldson H. Why do we not use trained interpreters for all patients with limited English proficiency? Is there a place for using family members? Australian Journal of Primary Health. 2011;17(3):240-9.

28. Amra S, O'Horo JC, Singh TD, Wilson GA, Kashyap R, Petersen R, et al. Derivation and validation of the automated search algorithms to identify cognitive impairment and dementia in electronic health records. Journal of critical care. 2017;37:202-5.

29. Guru PK, Singh TD, Passe M, Kashani KB, Schears GJ, Kashyap R. Derivation and validation of a search algorithm to retrospectively identify CRRT initiation in the ECMO patients. Applied clinical informatics. 2016;7(2):596.

30. 2006 Minnesota Statutes. Section 144.335. https://wwwrevisormngov/statutes/2006/cite/144335.

31. Herasevich V, Pickering BW, Dong Y, Peters SG, Gajic O, editors. Informatics infrastructure for syndrome surveillance, decision support, reporting, and modeling of critical illness. Mayo Clinic Proceedings; 2010: Elsevier.

32. Singh B, Singh A, Ahmed A, Wilson GA, Pickering BW, Herasevich V, et al., editors. Derivation and validation of automated electronic search strategies to extract Charlson comorbidities from electronic medical records. Mayo Clinic Proceedings; 2012: Elsevier.

33. Alsara A, Warner DO, Li G, Herasevich V, Gajic O, Kor DJ, editors. Derivation and validation of automated electronic search strategies to identify pertinent risk factors for postoperative acute lung injury. Mayo Clinic Proceedings; 2011: Elsevier.

34. Medicare Cf, Medicaid Services H. Medicare and Medicaid programs; electronic health record incentive program. Final rule. Federal register. 2010;75(144):44313.

35. Sittig DF, Singh $\mathrm{H}$. Electronic health records and national patient-safety goals. The New England journal of medicine. 2012;367(19):1854.

36. Kruse CS, Mileski M, Alaytsev V, Carol E, Williams A. Adoption factors associated with electronic health record among long-term care facilities: a systematic review. BMJ open. 2015;5(1).

37. Milinovich A, Kattan MW. Extracting and utilizing electronic health data from Epic for research. Annals of translational medicine. 2018;6(3).

38. Johnson R. A comprehensive review of an electronic health record system soon to assume market ascendancy: EPIC. J Healthc Commun. 2016;1(4):36.

39. Cooper LA, Hill MN, Powe NR. Designing and evaluating interventions to eliminate racial and ethnic disparities in health care. Journal of general internal medicine. 2002;17(6):477-86. 
40. Smischney NJ, Velagapudi VM, Onigkeit JA, Pickering BW, Herasevich V, Kashyap R. Derivation and validation of a search algorithm to retrospectively identify mechanical ventilation initiation in the intensive care unit. BMC Medical Informatics and Decision Making. 2014;14(1):1-6.

41. Rosenberg E, Seller R, Leanza Y. Through interpreters' eyes: comparing roles of professional and family interpreters. Patient education and counseling. 2008;70(1):87-93.

42. Bernstein J, Bernstein E, Dave A, Hardt E, James T, Linden J, et al. Trained medical interpreters in the emergency department: effects on services, subsequent charges, and follow-up. Journal of immigrant health. 2002;4(4):171-6.

43. Juckett G, Unger K. Appropriate use of medical interpreters. American Family Physician. 2014;90(7):476-80.

44. Blake C. Ethical considerations in working with culturally diverse populations: the essential role of professional interpreters. Bull Can Psychiatric Assoc. 2003;34:21-3.

45. Rollins G. Translation, por favor. Hospitals \& Health Networks. 2002;76(12):46-50, 1.

\section{Figures}




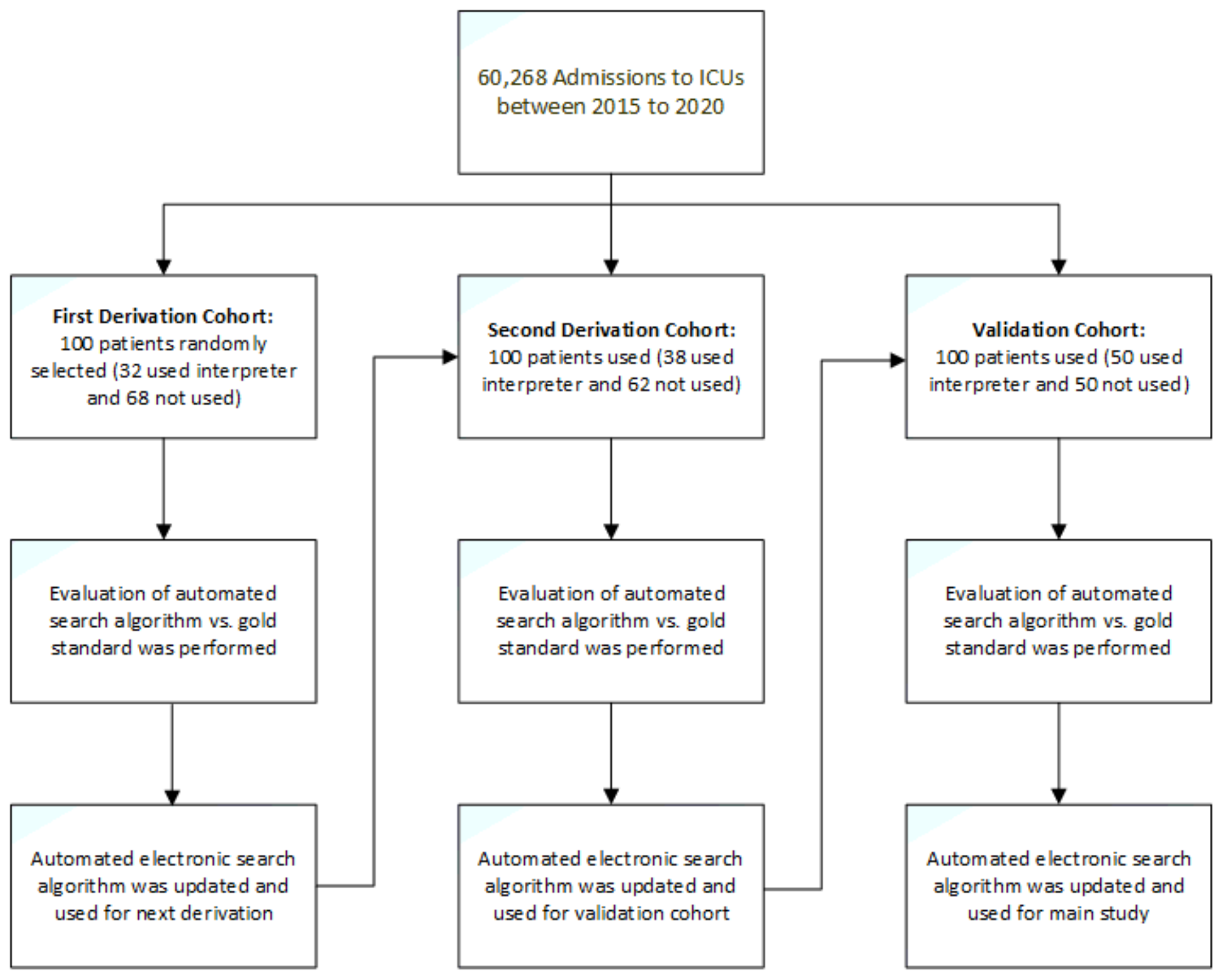

\section{Figure 1}

Enrollment of admissions to derivations and validation cohorts 